The Association

ACAMI for Child and Adolescent

Mental Health 


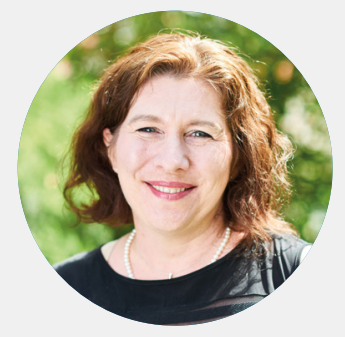

\section{Dr Juliette Kennedy}

\section{The Bridge Editor}

I am Dr. Juliette Kennedy, Editor of The Bridge, and a Consultant Child and Adolescent Psychiatrist working clinically in a North Yorkshire CAMHS team. I am Associate Director of Medical Education in the trust I work in, also Training Program director for CAMHS higher training in Yorkshire.

The Bridge presents the most clinically-relevant research from our two peer-reviewed journals: Child and Adolescent Mental Health and The Journal of Child Psychology and Psychiatry, as well as interesting and important studies from the wider literature. Please let us know what you'd like to see in upcoming editions by sending an email to me at: researchdigests@acamh.org

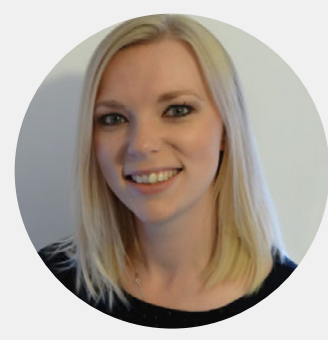

\section{Dr Jessica K. Edwards}

Research highlights in this edition are prepared by $\mathrm{Dr}$ Jessica K. Edwards. Jessica is a freelance editor and science writer, and started writing for 'The Bridge' in December 2017.

\section{Contents:}

p3 Child mental health professionals need more technology training

p4 Assessing and treating anxiety disorders: does one size really fit all?

p6 Can 'FRIENDS' in school help prevent anxiety?

P7 Obsessive compulsive disorder symptoms predict anxiety, and vice versa

p8 Can adult ASD outcomes be predicted by clinical measures made during childhood?

p9 Early adoption protects against internalizing, but not externalizing, problems

pil Is parental educational status to blame for academic problems in children? 



\title{
Assessing and treating anxiety disorders: does one size really fit all?
}

\author{
By Jessica K. Edwards
}

Cathy Creswell, Polly Waite and Jennie Hudson have written the first Practitioner Review for JCPP in 2020. The researchers first describe how anxiety disorders might develop and be maintained, and then focus on the various assessment and treatment considerations for children and adolescents with anxiety disorders.

They first explain that anxiety disorders should be routinely assessed using valid and reliable interviewbased and questionnaire-based measures of anxiety which also detect common co-morbid problems. To obtain accurate differential diagnoses, mood and behaviour disorders and the risk of suicide and selfinjury, should be assessed. To ensure that clinicians fairly balance caregiver and self reports, Creswell et al. recommend that clinicians use the 'OR rule' proposed by Comer \& Kendall. ${ }^{1}$ Here, clinicians should include clinically interfering symptoms that are reported by either the young person or the parent when constructing the diagnostic profile.

Moving on to treatment, cognitive behavioural therapy (CBT) for anxiety has consistently shown substantial benefits over waitlist controls across various metaanalyses. ${ }^{2}$ Unfortunately, many have found that the relapse rate after CBT is high. ${ }^{3}$ In addition, Creswell and colleagues point out that we still do not fully understand which components of CBT programmes are the most effective. The data available thus far suggest that exposure tasks might accelerate improvements in symptom severity and global functioning, while relaxation training has a lesser effect. 4
According to Creswell et al., we should now identify precisely which strategies promote new learning through exposure, in young people, across the different stages of childhood development.

Worryingly, Creswell et al. highlight the extremely low rate of service use by affected children and adolescents in many countries, including the UK, USA and Australia. One study found that as few as $2 \%$ of children with anxiety disorders receive CBT. It thus seems that tools are urgently needed to help identify young people who might benefit from professional support to overcome anxiety problems. The researchers suggest that a stepped care model might help increase access to evidence-based interventions. Here, the least costly intervention is delivered initially; those who do not respond to this first step, move on to a more intensive intervention. ${ }^{6}$ The researchers explain that work is now needed to refine the stepped care model before it can be rolled out. Specifically, we need to (i) maximise the cost-effectiveness of each step, (ii) identify the optimal staff and setting and (iii) clarify the needs of children and adolescents who do not benefit from the first step.

Overall, it seems that while the prevalence of anxiety disorders in young people is increasing, access and uptake of evidence-based treatments remains low. Creswell and colleagues thus propose a number of lines of research that should be undertaken to improve on the current situation. For example, experimental studies are needed to determine the maintenance mechanisms of anxiety disorders and how they might change across childhood development. Large studies are also needed that examine the predictors, mediators and moderators of treatment to help optimize treatment outcomes. Ultimately, the hope is that treatment approaches move away from a "one-size-fits-all" model and that personalized care strategies are developed.
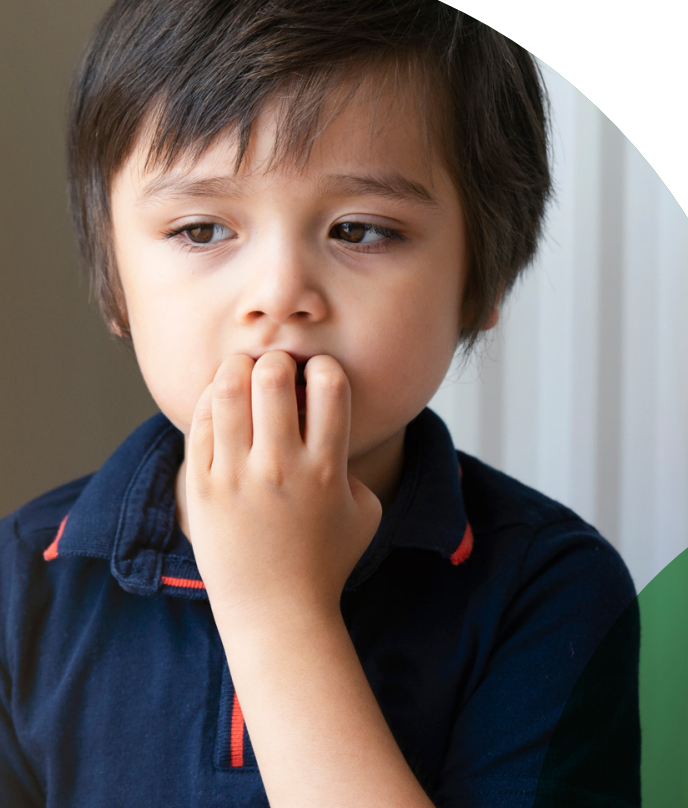




\title{
Can adult ASD outcomes be predicted by clinical measures made during childhood?
}

\author{
By Jessica K. Edwards
}

A new study has retrospectively profiled the adult outcomes of 123 children with autism spectrum disorder (ASD) to determine whether certain outcomes, or "types", can be predicted from typical clinical measures made during childhood. The researchers used a set of 15 variables to characterise adult outcomes, including the verbal and non-verbal IQ level, ASD symptom severity score, behavioural problems and medication use. From these, they found that only four classes of adult outcomes existed in their population: one good, one intermediate and two low-functioning classes (in which one had co-occurring behavioural problems). These classes could be confidently predicted from childhood verbal IQ levels and autism symptom severity recorded from age two to nine years. The accuracy of the prediction improved over time. Interestingly, removing verbal and non-verbal IQ and autism symptom severity measurements from the set of 15 variables had no effect on the number of the latent classes; however, the class composition changed and the class prediction accuracy diminished.
The researchers suggest that these data might help clinicians develop goals with individuals with ASD and do forward planning with respect to transition into adult mental health services. They do, however, urge caution in using these four classes as an aetiological taxonomy. This typology gave weight to cognitive outcomes while outcomes concerning subjective well-being and the affective domain were poorly predicted from childhood measures. Future research is thus needed to understand how to balance subjective measures of well-being and objective measures of functioning to describe positive adaptations in adulthood.

\section{Referring to:}

Pickles, A., McCauley, J.B., Pepa, L.A., Huerta, M. \& Lord, C. (2020), The adult outcome of children referred for autism: typology and prediction from childhood. J. Child Psychol. Psychiatry. doi: 10.111/ jcpp.13180. 


\title{
Early adoption protects against internalizing, but not externalizing, problems
}

\author{
By Jessica K. Edwards
}

The effects of childhood adoption on adult outcomes are conflicting; the adoption of children from highrisk backgrounds can have protective effects, as evidenced by generally positive cognitive skills and school achievements. ${ }^{1}$ However, adopted children can show increased vulnerability to mental health problems during childhood and adulthood. ${ }^{2,3}$ This increased vulnerability might be due to experiencing adverse life events prior to adoption or to problems in the postadoption home environment. Now, researchers in the UK have used data from two groups of early-adopted individuals (from the 1958 and 1970 British birth cohorts) to comprehensively describe outcomes up to mid-life. They compared them with the outcomes of children who were raised with two biological parents (a "general population" group) and also to children who were born to single mothers but were not adopted (a "birth comparison" group). The adopted children shared similar early characteristics with the birth comparison group but were placed in more socially advantaged adoptive homes.
The researchers found few differences between the adopted groups and the general population group in terms of physical health and psychological well-being, but more favourable outcomes in these domains compared to the birth comparison group. The rates of adult externalizing behaviours (including adult convictions, smoking and problem drinking) in the adopted and birth comparison groups, however, were higher compared to the rates in the general population group. These data suggest that although adoption can have protective effects in terms of internalizing problems, it might not be protective in relation to externalizing problems. The data from this study suggested that prenatal adversity might contribute to these externalizing problems. However, more work is needed to better understand the early and late experiences that contribute to this more negative trajectory in at-risk children. 


\section{Referring to:}

Sehmi, R., Rushton, A., Pickles, A., Grant, M. \& Maughan, B. (2020), Infant domestic adoption: outcomes at mid-life. J. Child Psychol. Psychiatry. doi: 10.111/jcpp.13178.

\section{References:}

${ }^{1}$ van ljzendoorn, M.H. et al. (2005), Adoption is a successful natural intervention enhancing adopted children's IQ and school performance. Curr. Dir. Psychol. Sci. 14,: 326-330. doi: 10.1111/j.09637214.2005.00391.x.

${ }^{2}$ Juffer, F. et al. (2005), Behaviour problems and mental health referrals of international adoptees - A meta-analysis. JAMA. 293: 2501-2515. doi: 10.1001/jama.293.20.2501.

${ }^{3}$ Behle, A.E. et al. (2016), Psychiatric disorders and treatment in adoptees: A meta-analytic comparison with non-adoptees. Adopt. Q. 19: 284-306. doi: 10.1080/10926755.2016.1201708.

\section{Glossary:}

Internalizing problems: individuals with internalizing problems typically attempt to conceal their maladaptive emotions and cognitions. This internalizing approach can manifest as depression, withdrawal, low self-esteem, anxiety and/or loneliness. Some affected individuals might also exhibit suicidal behaviours.

Externalizing problems: individuals with externalizing problems exhibit their maladaptive thoughts and emotions externally. Characteristic behaviours include impulsivity, and antisocial or aggressive behaviours. Adult manifestations of externalizing problems can include alcohol-related or substance-related disorders. 
